International Journal of Applied Mathematics

Volume 28 No. $5 \quad 2015,477-494$

ISSN: $1311-1728$ (printed version); ISSN: 1314-8060 (on-line version)

doi: http://dx.doi.org/10.12732/ijam.v28i5.2

\title{
TRANSIENT THERMOELASTIC PROBLEM FOR TWO REGION CONCENTRIC CYLINDER WITH PERFECT THERMAL CONTACT AT THE INTERFACE
}

\author{
Arti S. Sewalkar ${ }^{1}$, Suchitra A. Meshram² \\ ${ }^{1,2}$ Department of Mathematics \\ P.G.T.D. RTM Nagpur University \\ Nagpur, INDIA
}

\begin{abstract}
This paper is a treatment of a transient thermoelastic contact problem in two region concentric solid cylinder with perfect thermal contact at the interface subjected to convectional boundary condition at the outer surface $r=b$. The solution meets the boundary condition on both the lateral surfaces.

In order to analyse the problem, dimensionless quantities are used to develop the analysis of temperature field. We have used the method of generalized integral transforms. The dimensionless displacements and stresses have been attained using Goodier's thermoelastic function $\phi$ and Love's function $L$.
\end{abstract}

AMS Subject Classification: 35A25, 74M99, 74K20

Key Words: concentric solid cylinders, perfect thermal contact at interface, dimensionless quantities, Goodier's thermoelastic function, Love's function

\section{Introduction}

Some interest has been shown in the solutions of contact problems involving the heating of solid surfaces such as J.R. Barber [3], [4], [5] considered indentation of semi-infinite solid in elastic half space. D.L. Elements and G.D. Toy [6] considered two contact problems in anisotropic thermoelasticity. Z.S. Olesiak [8] considered axially symmetric thermal contact stress for a micropolar elastic semi-space. O. Tamate et al. [10] considered problem of singularities at the ends

Received: May 30, 2015

(C) 2015 Academic Publications

${ }^{\S}$ Correspondence author 
of thin elastic stiffener bounded to a semi-infinite medium. R.D. KulchytskyZhyhailo et al. [7] studied problem on thermal contact of two axially symmetric elastic solids. A.A. Yevtushenko and R.D. Kulchytsky-Zhyhailo [11] studied approximation solution of thermoelastic contact problem with frictional heating in general case. H.H. Sherif and K.A. Helmy [9] considered two dimensional problem for a half space in magnetothermoelasticity with thermal relaxation. G. Bonfanti et al. [1] studied existence exponential stability and dynamic contact problem of two thermoelastic beams. G. Bonfanti et al. [2] studied energy decay for a thermoelastic contact problem involving heat transfer.

This paper presents the thermoelastic contact problem of two region concentric cylinder with perfect thermal contact at interface. The purpose of this paper is to study heat transfer and thermoelastic effect in concentric regions. The results presented here have numerous applications in engineering of rocket liners, nuclear reactors etc.

To attain the solution of this problem, following attempts are made:

1. The solution for temperature field is obtained using orthogonal expansion technique over two layer region and Fourier Transforms.

2. Dimensionless quantities are used.

3. Goodier's thermelastic potential function $\phi$ and Love's function $L$ is used to obtain solution for thermal displacements and stresses.

4. The solution of the problem is presented in terms of dimensionless temperature, dimensionless displacements and dimensionless stresses for two regions, graphically represented and conclusions are made accordingly.

\section{Statement of the Heat Conduction Problem}

Consider a two region solid cylinder of outer radius $r=b$ with an inner radius $r=a$ and there is a perfect thermal contact between two layers. The cylinders are initially at uniform temperature $T_{0}$ and for times $t \geq 0$ the boundary surface at $r=b$ dissipates heat by the convection into a surrounding at zero. There is no heat generation within the solid body under consideration.

The two-dimensional transient heat conduction equations are given as

$$
\frac{\partial^{2} T_{1}}{\partial r^{2}}+\frac{1}{r} \frac{\partial T_{1}}{\partial r}+\frac{\partial^{2} T_{1}}{\partial z^{2}}=\frac{1}{\alpha_{1}} \frac{\partial T_{1}}{\partial t} \quad \text { in } 0 \leq r \leq a, t>0
$$




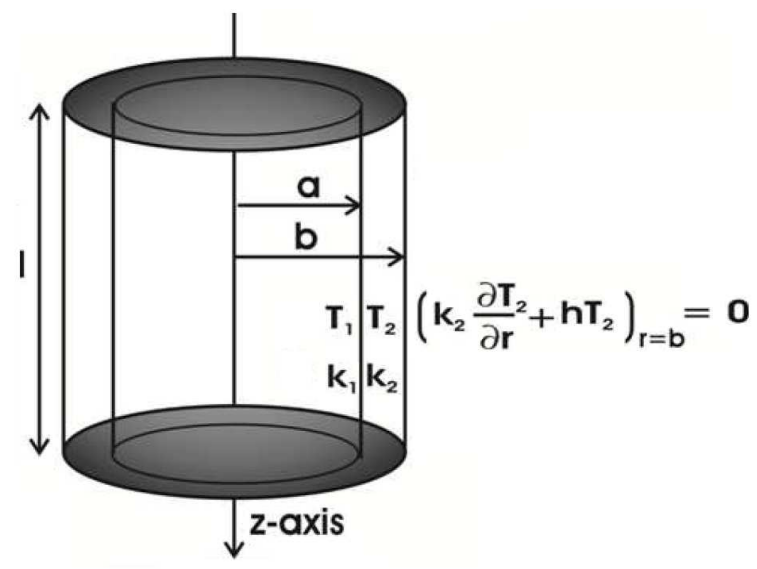

Figure 1: Shows the geometry of the problem

$$
\frac{\partial^{2} T_{2}}{\partial r^{2}}+\frac{1}{r} \frac{\partial T_{2}}{\partial r}+\frac{\partial^{2} T_{2}}{\partial z^{2}}=\frac{1}{\alpha_{2}} \frac{\partial T_{2}}{\partial t} \quad \text { in } a \leq r \leq b, t>0
$$

subject to boundary conditions

$$
\begin{gathered}
T_{1}=T_{2}=\operatorname{Taf}(z) \quad \text { at interface } r=a, t>0 \\
K_{1} \frac{\partial T_{1}}{\partial r}=K_{2} \frac{\partial T_{2}}{\partial r} \quad \text { at interface } r=a, t>0 \\
K_{2} \frac{\partial T_{2}}{\partial r}+h T_{2}=0 \quad \text { at the outer boundary condition } r=b, t>0
\end{gathered}
$$

with initial conditions

$$
\begin{gathered}
T_{1}=T_{0}=0 \text { in } 0 \leq r \leq a, t=0 \\
T_{2}=T_{0}=0 \quad \text { in } a \leq r \leq b, t=0,
\end{gathered}
$$

where $T_{0}$ is the initial temperature, $K_{1}, K_{2}$ be the thermal conductivities of the inner and outer region respectively, and $\alpha_{1}$ and $\alpha_{2}$ be the thermal diffusivities. Moreover, we use the following dimensionless quantities:

$$
\widehat{T}=\frac{T}{T_{a}}, \quad t^{6}=\frac{k t}{r_{i}^{2}}, \quad \rho=\frac{r}{r_{i}}, \quad \xi=\frac{z}{r_{i}} .
$$

Now we consider axi-symmetrical thermoelastic problem in a two region solid circular cylinder by use of Love's function $L$ and thermoelastic displacement potential $\phi$. The displacement and stresses are given by

$$
u_{\rho}=\frac{1}{r_{i}} \frac{\partial \phi_{i}}{\partial \rho}-\frac{1}{r_{i}^{2}} \frac{\partial L_{i}}{\partial \rho \partial \xi}
$$




$$
\begin{aligned}
u_{\xi} & =\frac{1}{r_{i}} \frac{\partial \phi_{i}}{\partial \xi}+2(1-\nu) \nabla^{2} L_{i}-\frac{1}{r_{i}^{2}} \frac{\partial^{2} L_{i}}{\partial \xi^{2}} \\
\frac{\bar{\rho} \bar{\rho}}{2 G} & =\frac{1}{r_{i}^{2}} \frac{\partial^{2} \phi_{i}}{\partial \rho^{2}}-\nabla^{2} \phi_{i}+\frac{1}{r_{i}} \frac{\partial\left(\nu \nabla^{2} L_{i}-\frac{1}{r_{i}^{2}} \frac{\partial^{2} L_{i}}{\partial \rho^{2}}\right)}{\partial \xi} \\
\frac{\bar{\theta} \bar{\theta}}{2 G} & =\frac{1}{\rho r_{i}^{2}} \frac{\partial \phi_{i}}{\partial \rho}-\nabla^{2} \phi_{i}+\frac{1}{r_{i}} \frac{\partial\left(\nu \nabla^{2} L_{i}-\frac{1}{\rho r_{i}^{2}} \frac{\partial L_{i}}{\partial \rho}\right)}{\partial \xi} \\
\frac{\bar{\xi} \bar{\xi}}{2 G} & =\frac{1}{r_{i}^{2}} \frac{\partial^{2} \phi_{i}}{\partial \xi^{2}}-\nabla^{2} \phi_{i}+\frac{1}{r_{i}} \frac{\partial\left((2-\nu) \nabla^{2} L_{i}-\frac{1}{r_{i}^{2}} \frac{\partial^{2} L_{i}}{\partial \xi^{2}}\right)}{\partial \xi} \\
\frac{\bar{\rho} \bar{\xi}}{2 G} & =\frac{1}{r_{i}^{2}} \frac{\partial^{2} \phi_{i}}{\partial \rho \partial \xi}+\frac{1}{r_{i}} \frac{\partial\left((1-\nu) \nabla^{2} L_{i}-\frac{1}{r_{i}^{2}} \frac{\partial^{2} L_{i}}{\partial \xi^{2}}\right)}{\partial \xi},
\end{aligned}
$$

where $i=1,2, G$ and $\nu$ are the shear modulus and Poisson's ratio respectively, and $\mu \rho$ and $\mu \xi$ are the displacements in the $\rho$ and $\xi$ directions, respectively.

The functions $L$ and $\phi$ must satisfy the following differential equations:

$$
\nabla^{2} \phi_{i}=\frac{(1+\nu)}{(1-\nu)} \alpha T_{i}, \quad \nabla^{4} L_{i}=0 .
$$

\section{Determination of Temperature Field}

The temperature suitable to this problem with prescribed and initial and boundary conditions is given by

$$
\begin{aligned}
T_{i}(r, z, t)=A_{0}+\int_{0}^{\infty} A(s) & \left(I_{0}(s r)-\sum_{n=1}^{\infty} \frac{A_{n} X_{j n}(r)}{\alpha_{j}\left(s_{m}^{2}+\beta_{j n}^{2}\right)}\right) \\
& \times\left(1-e^{-a_{j}}\left(s_{m}^{2}+\beta_{j n}^{2}\right) t\right) \cos s z d s, \quad t>0
\end{aligned}
$$

where $j=1,2, A_{0}$ and $A(s)$ are unknown functions and $I_{0}(\mathrm{sr})$ denotes the modified Bessel function of order zero.

Using initial and boundary conditions we obtained the temperature function as

$$
T_{i}=\int_{0}^{\infty} \frac{\bar{f}(s)}{I_{0}(s a)}\left(I_{0}(s r)-\sum_{n=1}^{\infty} \frac{A_{n} X_{j n}(r)}{\alpha_{j}\left(s_{m}^{2}+\beta_{j n}^{2}\right)}\right)
$$




$$
\times\left(1-e^{-a_{j}}\left(s_{m}^{2}+\beta_{j n}^{2}\right) t\right) \cos s z d s, \quad t>0,
$$

where the eigenfunction $X_{j n}(\mathrm{r})$ for two layer problem under consideration is

$$
\begin{gathered}
X_{1 n}(r)=J_{0}\left(\beta_{1 n} r\right) \text { in layer 1, i.e. } 0 \leq r \leq a, \\
X_{2 n}(r)=B_{n}\left(J_{0}\left(\beta_{2 n} r\right)\right)+C_{n} Y_{0}\left(\beta_{2 n} r\right) \text { in layer 2, i.e. } a \leq r \leq b .
\end{gathered}
$$

The function $Y_{0}\left(\beta_{2 n} r\right)$ is excluded from the solution for region 1 because it includes the origin.

The eigenvalues $\beta_{1 n}$ and $\beta_{2 n}$ are related by

$$
\alpha_{1} \beta_{1 n}^{2}=\alpha_{2} \beta_{2 n}^{2} .
$$

Now unknown $B_{n}, C_{n}$ and the eigenvalues $\beta_{j n}$ can be obtained using boundary conditions (3), (4) and (5).

Using the boundary conditions (4), $C_{n}$ can be obtained as

$$
C_{n}=\frac{h_{2}\left(J_{0}\left(\beta_{2 n} b\right)\right)-K_{2} \beta_{2 n} J_{1}\left(\beta_{2 n} b\right)}{K_{2} \beta_{2 n}\left(Y_{1}\left(\beta_{2 n} b\right)\right)-h_{2} Y_{0}\left(\beta_{2 n} b\right)} .
$$

Using the boundary condition (3) we obtain $B_{n}$ as

$$
B_{n}=\frac{J_{0}\left(\beta_{1 n} a\right)}{\left(J_{0}\left(\beta_{2 n} a\right)\right)+C_{n} Y_{0}\left(\beta_{2 n} a\right)} .
$$

Now $A_{n}$ can be obtained using the relation

$$
A_{n}=\frac{\sum_{i=1}^{\infty} W_{i}^{2} \int_{\text {layeri }} F(x) X_{i n}(x) W(x) d x}{\sum_{i=1}^{\infty} W_{i}^{2} \int_{\text {layeri }} X_{\text {in }}^{2}(x) W(x) d x},
$$

where

$$
\begin{gathered}
W_{i}^{2}=\frac{k_{1}}{\alpha_{1}}, \quad W=r, \quad F_{1}=T_{0}, \quad i=1,2, \\
X_{1 n}(r)=J_{0}\left(\beta_{1 n} r\right), \quad X_{2 n}(r)=B_{n}\left[\left(J_{0}\left(\beta_{2 n} r\right)\right)+C_{n} Y_{0}\left(\beta_{2 n} r\right)\right],
\end{gathered}
$$

we obtain $A_{n}$ using the above equations as

$$
\begin{gathered}
A_{n}=\frac{\frac{K_{1}}{\alpha_{1}} \int_{0}^{a} T_{0} J_{0}\left(\beta_{1 n} r\right) r d r+\frac{K_{2}}{\alpha_{2}} \int_{a}^{b} T_{0} B_{n}\left[J_{0}\left(\beta_{2 n} r\right)+C_{n} Y_{0}\left(\beta_{2 n} r\right)\right] r d r}{\frac{K_{1}}{\alpha_{1}} \int_{0}^{a} J_{0}^{2}\left(\beta_{1 n} r\right) r d r+\frac{K_{2}}{\alpha_{2}} \int_{a}^{b} B_{n}^{2}\left[J_{0}\left(\beta_{2 n} r\right)+C_{n} Y_{0}\left(\beta_{2 n} r\right)\right]^{2} r d r}, \\
A_{n}=\frac{2 T_{0}}{\alpha \beta_{1 n}}\left[J_{1}\left(\beta_{1 n} a\right)+\frac{K_{2}}{K_{1}} \sqrt{\frac{\alpha_{1}}{\alpha_{2}}}\left[\frac { b } { a } \left\{\left[B_{n} J_{1}\left(\beta_{2 n} b\right)+C_{n} Y_{1}\left(\beta_{2 n} b\right) r\right]\right.\right.\right.
\end{gathered}
$$




$$
\begin{gathered}
\left.\left.-\left[B_{n} J_{1}\left(\beta_{2 n} a\right)+C_{n} Y_{1}\left(\beta_{2 n} a\right)\right]\right\}\right]\left[J_{0}^{2}\left(\beta_{1 n} a\right)+J_{1}^{2}\left(\beta_{1 n} a\right)\right. \\
+\frac{K_{2}}{K_{1}} \frac{\alpha_{1}}{\alpha_{2}}\left[\frac{b^{2}}{a^{2}} \times\left\{\left[B_{n}\left(J_{0}\left(\beta_{2 n} b\right)+C_{n} Y_{0}\left(\beta_{2 n} b\right)\right)\right]^{2}\right.\right. \\
\left.+\left[B_{n}\left(J_{1}\left(\beta_{2 n} b\right)+C_{n} Y_{1}\left(\beta_{2 n} b\right)\right)\right]^{2}\right\}-\left\{\left[B_{n}\left(J_{0}\left(\gamma_{n} a\right)+C_{n} Y_{0}\left(\gamma_{n} a\right)\right)\right]^{2}\right. \\
\left.\left.\left.+\left[B_{n}\left(J_{1}\left(\beta_{2 n} a\right)+C_{n} Y_{1}\left(\beta_{2 n} a\right)\right)\right]^{2}\right\}\right]\right]^{-1},
\end{gathered}
$$

and the finite Fourier Transform $T_{a} f(z)$ is defined as

$$
\bar{f}(s)=\int_{0}^{l} T_{a} f(z) \cos s z d z .
$$

Now the temperatures for different layers (i.e. $0 \leq r \leq a$ as layer 1 and $a \leq$ $r \leq b$ as layer 2) are:

$$
\begin{aligned}
T_{1}(r, z, t)=\sum_{m=1}^{\infty} \frac{T_{a} \bar{f}\left(s_{m}\right)}{I_{0}\left(s_{m} a\right)}\left[I_{0}\left(s_{m} r\right)\right. & \left.-\sum_{n=1}^{\infty} \frac{A_{n} J_{0}\left(\beta_{1 n} r\right)}{\alpha_{1}\left(s_{m}^{2}+\beta_{1 n}^{2}\right)}\right] \\
& \times\left(1-e^{-\alpha_{1}}\left(s_{m}^{2}+\beta_{1 n}^{2}\right) t\right) \cos s_{m} z, \quad \\
T_{2}(r, z, t)=\sum_{m=1}^{\infty} \frac{T_{a} \bar{f}\left(s_{m}\right)}{I_{0}\left(s_{m} a\right)}\left[I_{0}\left(s_{m} r\right)-\right. & \left.\sum_{n=1}^{\infty} \frac{A_{n}\left[B_{n}\left(J_{0}\left(\beta_{2 n} r\right)+C_{n} Y_{0}\left(\beta_{2 n} r\right)\right)\right]}{\alpha_{2}\left(s_{m}^{2}+\beta_{2 n}^{2}\right)}\right] \\
& \times\left(1-e^{-a_{2}}\left(s_{m}^{2}+\beta_{2 n}^{2}\right) t\right) \cos s_{m} z .
\end{aligned}
$$

Using the dimensionless quantities from (8) in equations (20) and (21), we obtain dimensionless temperature for two layers (i.e. $0 \leq \rho \leq a$ as layer 1 , and $a \leq \rho \leq b$ as layer 2 ) as

$$
\begin{aligned}
T_{1}\left(\rho, \xi, t^{\prime}\right)=\sum_{m=1}^{\infty} \frac{\bar{f}\left(s_{m}\right)}{I_{0}\left(s_{m} a\right)}\left[I_{0}\left(s_{m} \rho a\right)\right. & \left.-\sum_{n=1}^{\infty} \frac{A_{n} J_{0}\left(\beta_{1 n} \rho a\right)}{\alpha_{1}\left(s_{m}^{2}+\beta_{1 n}^{2}\right)}\right] \\
& \times\left(1-e^{-\alpha_{1}\left(s_{m}^{2}+\beta_{1 n}^{2}\right) \frac{a^{2} t^{\prime}}{K}}\right) \cos s_{m} \xi a, \quad(22) \\
T_{2}\left(\rho, \xi, t^{\prime}\right)=\sum_{m=1}^{\infty} \frac{\bar{f}\left(s_{m}\right)}{I_{0}\left(s_{m} a\right)}\left[I_{0}\left(s_{m} \rho b\right)\right. & \left.-\sum_{n=1}^{\infty} \frac{A_{n}\left[B_{n}\left(J_{0}\left(\beta_{2 n} \rho b\right)+C_{n} Y_{0}\left(\beta_{2 n} \rho b\right)\right)\right]}{\alpha_{2}\left(s_{m}^{2}+\beta_{2 n}^{2}\right)}\right] \\
& \times\left(1-e^{-a_{2}\left(s_{m}^{2}+\beta_{2 n}^{2}\right) \frac{b^{2} t^{\prime}}{K}}\right) \cos s_{m} \xi b . \quad(23)
\end{aligned}
$$




\section{Determination of Displacement Potential and Love's function}

Using equations (22) and (23) in equation (15), the displacement potential and Love's function is given as

$$
\begin{aligned}
\nabla^{2} \phi= & \frac{1+\nu}{1-\nu} \alpha T \\
\phi_{1}= & \frac{1+\nu}{1-\nu} \alpha_{1}^{2} \frac{a^{2}}{K} T_{a} \sum_{m=1}^{\infty} \frac{\bar{f}\left(s_{m}\right)}{I_{0}\left(s_{m} a\right)}\left[I_{0}\left(s_{m} \rho a\right)-\sum_{n=1}^{\infty} \frac{A_{n} J_{0}\left(\beta_{1 n} \rho a\right)}{\alpha_{1}\left(s_{m}^{2}+\beta_{1 n}^{2}\right)}\right] \\
& \times\left(t^{\prime}+\frac{e^{-\alpha_{1}}\left(s_{m}^{2}+\beta_{1 n}^{2}\right) \frac{a^{2} t^{1}}{K}}{\alpha_{1}\left(s_{m}^{2}+\beta_{1 n}^{2}\right) \frac{a^{2}}{K}}\right) \cos s_{m} \xi a .
\end{aligned}
$$

Similarly,

$$
\begin{aligned}
\phi_{2}= & \frac{1+\nu}{1-\nu} \alpha_{2}^{2} \frac{b^{2}}{K} T_{a} \sum_{m=0}^{\infty} \frac{\bar{f}(s)}{I_{0}\left(s_{m} a\right)} \\
& \times\left[I_{0}\left(s_{m} \rho b\right)-\sum_{n=1}^{\infty} \frac{A_{n}}{\alpha_{2}\left(s_{m}^{2}+\beta_{2 n}^{2}\right)}\left[B_{n}\left[J_{0}\left(\beta_{2 n} \rho b\right)+C_{n} Y_{0}\left(\beta_{2 n} \rho b\right)\right]\right]\right] \\
& \times\left(t^{\prime}+\frac{e^{-\alpha_{2}\left(s_{m}^{2}+\beta_{2 n}^{2}\right) \frac{b^{2} t^{1}}{K}}}{\alpha_{2}\left(s_{m}^{2}+\beta_{2 n}^{2}\right) \frac{b^{2}}{K}}\right) \cos s_{m} \xi b
\end{aligned}
$$

Now Love's function is given as

$$
\begin{aligned}
L_{1}=\frac{1+\nu}{1-\nu} \alpha_{1} & \frac{a^{2}}{K} T_{a} \\
& \times \sum_{m=1}^{\infty} \frac{\bar{f}\left(s_{m}\right)}{s_{m}^{2}}\left[D_{n} I_{0}\left(s_{m} \rho a\right)+E_{n} s_{m} \rho a I_{1}\left(s_{m} \rho a\right)\right] \sin s_{m} \xi a
\end{aligned}
$$

and

$$
\begin{aligned}
L_{2}=\frac{1+\nu}{1-\nu} \alpha_{2} & \frac{b^{2}}{K} T_{a} \\
& \times \sum_{m=1}^{\infty} \frac{\bar{f}\left(s_{m}\right)}{s_{m}^{2}}\left[D_{n} I_{0}\left(s_{m} \rho b\right)+E_{n} s_{m} \rho b I_{1}\left(s_{m} \rho b\right)\right] \sin s_{m} \xi b .
\end{aligned}
$$




\section{Determination of Thermal Displacements and Stresses for Region 1}

Using equations (24) and (26) in equations (9) to (10), the thermal displacements $U_{\rho}$ are given as

$$
\begin{aligned}
U_{\rho}= & \frac{1+\nu}{1-\nu} \alpha_{1} \frac{a^{2}}{K} T_{a} \sum_{m=0}^{\infty} \frac{\bar{f}\left(s_{m}\right)}{I_{0}\left(s_{m} a\right)} \\
& \times\left[I_{0}^{\prime}\left(s_{m} \rho a\right) s_{m}-\sum_{n=1}^{\infty} \frac{A_{n}}{\alpha_{1}\left(s_{m}^{2}+\beta_{1 n}^{2}\right)} J_{0}^{\prime}\left(\beta_{1 n} \rho a\right) \beta_{1 n} a\right] \\
& \times\left(t^{\prime}+\frac{e^{-\alpha_{1}\left(s_{m}^{2}+\beta_{1 n}^{2}\right) \frac{a^{2} t^{\prime}}{K}}}{\alpha_{1}\left(s_{m}^{2}+\beta_{1 n}^{2}\right) \frac{a^{2}}{K}}\right) \cos s_{m} \xi a \\
& -\frac{1+\nu}{1-\nu} \frac{\alpha_{1}}{K} T_{a} \sum_{m=0}^{\infty} \frac{\bar{f}\left(s_{m}\right)}{s_{m}^{2}} \\
& \times\left[D_{n} I_{0}^{\prime}\left(s_{m} \rho a\right) s_{m} a+E_{n} s_{m} \rho I_{1}^{\prime}\left(s_{m} \rho a\right) s_{m} a+I_{1}\left(s_{m} \rho a\right) s_{m} a\right] \\
& \times \cos s_{m} \xi a .
\end{aligned}
$$

Similarly, we can get thermal displacement $U_{\xi}$.

Using equations (24) and (26) in equations (11) to (14), the thermal stresses are given as

$$
\begin{aligned}
\frac{\bar{\rho} \bar{\rho}}{2 G}= & \frac{1+\nu}{1-\nu} \alpha_{1} \frac{a^{2}}{K} T_{a} \\
& \times \sum_{m=0}^{\infty} \frac{\bar{f}\left(s_{m}\right)}{I_{0}\left(s_{m} a\right)}\left[I_{0}^{\prime \prime}\left(s_{m} \rho a\right) s_{m}^{2}\right. \\
& \left.-\sum_{n=1}^{\infty} \frac{A_{n}}{\alpha_{1}\left(s_{m}^{2}+\beta_{1 n}^{2}\right)} J_{0}^{\prime \prime}\left(\beta_{1 n} \rho a\right) \beta_{1 n}^{2}\right] \\
& \times\left(t^{\prime}+\frac{e^{-\alpha_{1}\left(s_{m}^{2}+\beta_{1 n}^{2}\right) \frac{a^{2} t^{\prime}}{K}}}{\alpha_{1}\left(s_{m}^{2}+\beta_{1 n}^{2}\right) \frac{a^{2}}{K}}\right) \cos s_{m} \xi a \\
& -\left\{\frac{1+\nu}{1-\nu} \alpha_{1} \frac{a^{2}}{K} T_{a} \sum_{m=0}^{\infty} \frac{\bar{f}\left(s_{m}\right)}{I_{0}\left(s_{m} a\right)}\left[I_{0}^{\prime \prime}\left(s_{m} \rho a\right) s_{m}^{2}\right]\right. \\
& \left.-\sum_{n=1}^{\infty} \frac{A_{n}}{\alpha_{1}\left(s_{m}^{2}+\beta_{1 n}^{2}\right)} J_{0}^{\prime \prime}\left(\beta_{1 n} \rho a\right) \beta_{1 n}^{2}\right]
\end{aligned}
$$




$$
\begin{aligned}
& \times\left(t^{\prime}+\frac{e^{-\alpha_{1}\left(s_{m}^{2}+\beta_{1 n}^{2}\right) \frac{a^{2} t^{\prime}}{K}}}{\alpha_{1}\left(s_{m}^{2}+\beta_{1 n}^{2}\right) \frac{a^{2}}{K}}\right) \cos s_{m} \xi a \\
& +\frac{1}{\rho} \frac{1+\nu}{1-\nu} \alpha_{1} \frac{a}{K} T_{a} \sum_{m=0}^{\infty} \frac{\bar{f}\left(s_{m}\right)}{I_{0}\left(s_{m} a\right)}\left[I_{0}^{\prime}\left(s_{m} \rho a\right) s_{m}^{2}\right. \\
& \left.-\sum_{n=1}^{\infty} \frac{A_{n}}{\alpha_{1}\left(s_{m}^{2}+\beta_{1 n}^{2}\right)} J_{0}^{\prime}\left(\beta_{1 n} \rho a\right) \beta_{1 n}^{2}\right] \\
& \times\left(t^{\prime}+\frac{e^{-\alpha_{1}\left(s_{m}^{2}+\beta_{1 n}^{2}\right) \frac{a^{2} t^{\prime}}{K}}}{\alpha_{1}\left(s_{m}^{2}+\beta_{1 n}^{2}\right) \frac{a^{2}}{K}}\right) \cos s_{m} \xi a \\
& -\frac{1+\nu}{1-\nu} \alpha_{1} \frac{a^{2}}{K} T_{a} \sum_{m=0}^{\infty} \frac{\bar{f}\left(s_{m}\right)}{I_{0}\left(s_{m} a\right)}\left[I_{0}\left(s_{m} \rho a\right) s_{m}^{2}\right. \\
& \left.-\sum_{n=1}^{\infty} \frac{A_{n}}{\alpha_{1}\left(s_{m}^{2}+\beta_{1 n}^{2}\right)} J_{0}\left(\beta_{1 n} \rho a\right)\right] \\
& \times\left(t^{\prime}+\frac{e^{-\alpha_{1}\left(s_{m}^{2}+\beta_{1 n}^{2}\right) \frac{a^{2} t^{\prime}}{K}}}{\alpha_{1}\left(s_{m}^{2}+\beta_{1 n}^{2}\right) \frac{a^{2}}{K}}\right)\left(\cos s_{m} \xi a\right) s_{m}^{2} \\
& +\left\{\frac { \nu ( 1 + \nu ) } { ( 1 - \nu ) } \alpha _ { 1 } \frac { a ^ { 2 } } { K } \sum _ { m = 0 } ^ { \infty } \overline { f } ( s _ { m } ) \left[D_{n} I_{0}^{\prime \prime}\left(s_{m} \rho a\right)\right.\right. \\
& \left.+\rho I_{1}^{\prime \prime}\left(s_{m} \rho a\right) s_{m} a+2 I^{\prime}\left(s_{m} \rho a\right)\right] \cos s_{m} \xi a s_{m} \\
& +\frac{1}{\rho} \frac{\nu(1+\nu)}{(1-\nu)} \alpha_{1} \frac{a}{K} T_{a} \sum_{m=0}^{\infty} \frac{\bar{f}\left(s_{m}\right)}{s_{m}^{2}}\left[D_{n} I_{0}^{\prime}\left(s_{m} \rho a\right) s_{m}\right. \\
& +E_{n} s_{m} \rho I_{1}^{\prime}\left(s_{m} \rho a\right) s_{m} a+I_{1}\left(s_{m} \rho a\right) \\
& -\frac{\nu(1+\nu)}{(1-\nu)} \alpha_{1} \frac{a^{2}}{K} T_{a} \sum_{m=0}^{\infty} \bar{f}\left(s_{m}\right)\left[D_{n} I_{0}\left(s_{m} \rho a\right)\right. \\
& \left.\left.+E_{n} s_{m} \rho a I_{1}\left(s_{m} \rho a\right)\right] \cos s_{m} \xi a s_{m}\right\} \\
& +\frac{(1+\nu)}{(1-\nu)} \alpha_{1} \frac{a^{2}}{K} T_{a} \sum_{m=0}^{\infty} \frac{\bar{f}\left(s_{m}\right)}{s_{m}^{2}}\left[D_{n} I_{0}^{\prime \prime}\left(s_{m} \rho a\right) s_{m}^{2}\right. \\
& \left.+E_{n} s_{m} \rho I_{1}^{\prime \prime}\left(s_{m} \rho a\right) s_{m}^{2}+I_{1}^{\prime}\left(s_{m} \rho a\right) s_{m}+I_{1}^{\prime}\left(s_{m} \rho a\right) s_{m}\right] \cos s_{m} \xi a s_{m}, \\
& \frac{\bar{\rho} \bar{\xi}}{2 G}=\frac{(1+\nu)}{(1-\nu)} \frac{\alpha_{1}}{K} T_{a}
\end{aligned}
$$




$$
\begin{aligned}
& \times \sum_{m=0}^{\infty} \frac{\bar{f}\left(s_{m}\right)}{I_{0}\left(s_{m} a\right)}\left[I_{0}^{\prime}\left(s_{m} \rho a\right) s_{m} a-\sum_{n=1}^{\infty} \frac{A_{n}}{\alpha_{1}\left(s_{m}^{2}+\beta_{1 n}^{2}\right) a^{2}} J_{0}^{\prime}\left(\beta_{1 n} \rho a\right)\right] \\
& \times\left(t^{\prime}+\frac{e^{-\alpha_{1}\left(s_{m}^{2}+\beta_{1 n}^{2}\right) \frac{a^{2} t^{\prime}}{K}}}{\alpha_{1}\left(s_{m}^{2}+\beta_{1 n}^{2}\right) \frac{a^{2}}{K}}\right) \sin \left(s_{m} \xi a\right) s_{m} a \\
& +\frac{(1+\nu)}{(1-\nu)} \alpha_{1} \frac{a}{K} T_{a} \sum_{m=0}^{\infty} \bar{f}\left(s_{m}\right)\left[D_{n} I_{0}^{\prime \prime}\left(s_{m} \rho a\right) s_{m} a\right. \\
& \left.+E_{n}\left\{I_{1}^{\prime \prime \prime}\left(s_{m} \rho a\right) s_{m} a^{2}+I_{1}^{\prime \prime}\left(s_{m} \rho a\right) s_{m} a+2 I_{1}^{\prime}\left(s_{m} \rho a\right) a\right\}\right] \sin \left(s_{m} \xi a\right) \\
& +\frac{1}{\rho} \frac{(1+\nu)}{(1-\nu)} \frac{\alpha_{1}}{K} T_{a} \sum_{m=0}^{\infty} \bar{f}\left(s_{m}\right)\left[D_{n} I_{0}^{\prime \prime}\left(s_{m} \rho a\right) a^{2}\right. \\
& \left.+E_{n}\left\{I_{1}^{\prime \prime}\left(s_{m} \rho a\right) s_{m} a^{2}+I_{1}^{\prime}\left(s_{m} \rho a\right) a+I_{1}^{\prime}\left(s_{m} \rho a\right) a\right\}\right] \sin \left(s_{m} \xi a\right) \\
& -\frac{1}{\rho^{2}} \frac{(1+\nu)}{(1-\nu)} \frac{\alpha_{1}}{K} T_{a} \sum_{m=0}^{\infty} \frac{\bar{f}\left(s_{m}\right)}{s_{m}}\left[D_{n} I_{0}^{\prime}\left(s_{m} \rho a\right) a\right. \\
& \left.+E_{n}\left\{\rho I_{1}^{\prime}\left(s_{m} \rho a\right) s_{m} a+I_{1}\left(s_{m} \rho a\right)\right\}\right] \sin \left(s_{m} \xi a\right) \\
& +\frac{(1+\nu)}{(1-\nu)} \frac{\alpha_{1}}{K} T_{a} \sum_{m=0}^{\infty} \bar{f}\left(s_{m}\right)\left[D_{n} I_{0}^{\prime}\left(s_{m} \rho a\right) s_{m} a\right. \\
& \left.+E_{n} s_{m}\left\{\rho a I_{1}^{\prime}\left(s_{m} \rho a\right) s_{m} a+a I_{1}\left(s_{m} \rho a\right)\right\}\right] \sin \left(s_{m} \xi a\right) \\
& +\frac{(1+\nu)}{(1-\nu)} \frac{\alpha_{1}}{K} T_{a} \sum_{m=0}^{\infty} \bar{f}\left(s_{m}\right)\left[D_{n} I_{0}^{\prime}\left(s_{m} \rho a\right) s_{m} a\right. \\
& \left.+E_{m} a\left\{\rho I_{1}^{\prime}\left(s_{m} \rho a\right) s_{m} a+I_{1}\left(s_{m} \rho a\right)\right\}\right] \sin \left(s_{m} \xi a\right) .
\end{aligned}
$$

Similarly we can obtain the stresses $\sigma_{\xi \xi}$ and $\sigma_{\theta \theta}$.

\section{Determination of Thermal Displacements and Stresses for Region 2}

Using equations (25) and (27) in equations (9) and (10), the thermal displacements for region 2 are given as

$$
\begin{aligned}
U_{\rho}= & \frac{(1+\nu)}{(1-\nu)} \frac{\alpha_{2}}{K} T_{a}\left\{\sum_{m=0}^{\infty} \frac{\bar{f}\left(s_{m}\right)}{I_{0}\left(s_{m} a\right)}\left[I_{0}^{\prime}\left(s_{m} \rho b\right) s_{m}\right]\right. \\
& -\sum_{n=1}^{\infty} \frac{A_{n}}{\alpha_{2}\left(s_{m}^{2}+\beta_{2 n}^{2}\right)}\left[B_{n}\left\{J_{0}^{\prime}\left(\beta_{2 n} \rho b\right) \beta_{2 n}+C_{n} Y_{0}^{\prime}\left(\beta_{2 n} \rho b\right)\right\} \beta_{2 n}\right]
\end{aligned}
$$




$$
\begin{aligned}
& \times\left(t^{\prime}+\frac{e^{-\alpha_{2}\left(s_{m}^{2}+\beta_{2 n}^{2}\right) \frac{b^{2} t^{\prime}}{K}}}{\alpha_{2}\left(s_{m}^{2}+\beta_{2 n}^{2}\right) \frac{b^{2}}{K}}\right) \cos \left(s_{m} \xi b\right) \\
& -\sum_{m=0}^{\infty} \frac{\bar{f}\left(s_{m}\right)}{s_{m}}\left[D_{n} I_{0}^{\prime}\left(s_{m} \rho b\right) s_{m} b\right. \\
& \left.\left.+E_{n} s_{m}\left\{\rho I_{1}^{\prime}\left(s_{m} \rho b\right) s_{m} b+I_{1}\left(s_{m} \rho b\right)\right\}\right] \cos s_{m} \xi b\right\} .
\end{aligned}
$$

Similarly we can get displacement $U_{\xi}$.

Using (27) and (30) in equations (11) to (14), the thermal stresses are given as

$$
\begin{aligned}
\frac{\bar{\rho} \bar{\rho}}{2 G}= & \frac{(1+\nu)}{(1-\nu)} b^{2} \frac{\alpha_{2}}{K} T_{a} \sum_{m=0}^{\infty} \frac{\bar{f}\left(s_{m}\right)}{I_{0}\left(s_{m} a\right)}\left[I_{0}^{\prime \prime}\left(s_{m} \rho b\right) s_{m}^{2}\right. \\
& \left.\sum_{n=1}^{\infty} \frac{A_{n}}{\alpha_{2}\left(s_{m}^{2}+\beta_{2 n}^{2}\right)}\left[B_{n}\left\{J_{0}^{\prime \prime}\left(\beta_{2 n} \rho b\right) \beta_{2 n}^{2}+C_{n} Y_{0}^{\prime \prime}\left(\beta_{2 n} \rho b\right)\right\} \beta_{2 n}^{2}\right]\right] \\
& \times\left(t^{\prime}+\frac{e^{-\alpha_{2}\left(s_{m}^{2}+\beta_{2 n}^{2}\right)} \frac{b^{2} t^{\prime}}{\alpha_{2}}}{\left.\alpha_{m}^{2}+\beta_{2 n}^{2}\right) \frac{b^{2}}{K}}\right) \cos s_{m} \xi b \\
& -\left\{\frac { ( 1 + \nu ) } { ( 1 - \nu ) } b ^ { 2 } \frac { \alpha _ { 2 } } { K } T _ { a } \sum _ { m = 0 } ^ { \infty } \frac { \overline { f } ( s _ { m } ) } { I _ { 0 } ( s _ { m } a ) } \left[I_{0}^{\prime \prime}\left(s_{m} \rho b\right) s_{m}^{2}\right.\right. \\
& \left.-\sum_{n=1}^{\infty} \frac{A_{n}}{\alpha_{2}\left(s_{m}^{2}+\beta_{2 n}^{2}\right)}\left[B_{n}\left\{J_{0}^{\prime \prime}\left(\beta_{2 n} \rho b\right) \beta_{2 n}^{2}+C_{n} Y_{0}^{\prime \prime}\left(\beta_{2 n} \rho b\right)\right\} \beta_{2 n}^{2}\right]\right] \\
& \times\left(t^{\prime}+\frac{e^{-\alpha_{2}\left(s_{m}^{2}+\beta_{2 n}^{2}\right) \frac{b^{2} t^{\prime}}{K}}}{\alpha_{2}\left(s_{m}^{2}+\beta_{2 n}^{2}\right) \frac{b^{2}}{K}}\right) \cos s_{m} \xi b \\
& +\frac{(1+\nu)}{(1-\nu)} \frac{\alpha_{2}}{\rho K} T_{a} \sum_{m=0}^{\infty} \frac{\bar{f}\left(s_{m}\right)}{I_{0}\left(s_{m} a\right)}\left[I_{0}^{\prime}\left(s_{m} \rho b\right) s_{m} b\right. \\
& \left.-\sum_{n=1}^{\infty} \frac{A_{n}}{\alpha_{2}\left(s_{m}^{2}+\beta_{2 n}^{2}\right)}\left[B_{n}\left\{J_{0}^{\prime}\left(\beta_{2 n} \rho b\right) \beta_{2 n} b+C_{n} Y_{0}^{\prime}\left(\beta_{2 n} \rho b\right)\right\} \beta_{2 n} b\right]\right] \\
& \times\left(t^{6}+\frac{e^{-\alpha_{2}\left(s_{m}^{2}+\beta_{2 n}^{2}\right.}}{\alpha_{2}\left(s_{m}^{2}+\beta_{2 n}^{2}\right) \frac{b^{2} t^{\prime}}{K}}\right) \cos s_{m} \xi b
\end{aligned}
$$




$$
\begin{aligned}
& -\frac{(1+\nu)}{(1-\nu)} \frac{\alpha_{2}}{K} T_{a} \sum_{m=0}^{\infty} \frac{\bar{f}\left(s_{m}\right)}{I_{0}\left(s_{m} a\right)}\left[\quad I_{0}\left(s_{m} \rho b\right)\right. \\
& \left.-\sum_{n=1}^{\infty} \frac{A_{n}}{\alpha_{2}\left(s_{m}^{2}+\beta_{2 n}^{2}\right)}\left[B_{n}\left\{J_{0}\left(\beta_{2 n} \rho b\right)+C_{n} Y_{0}\left(\beta_{2 n} \rho b\right)\right\}\right]\right] \\
& \times\left(t^{\prime}+\frac{e^{-\alpha_{2}\left(s_{m}^{2}+\beta_{2 n}^{2}\right) \frac{b^{2} t^{\prime}}{K}}}{\alpha_{2}\left(s_{m}^{2}+\beta_{2 n}^{2}\right) \frac{b^{2}}{K}}\right) \cos \left(s_{m} \xi b\right) s_{m} \\
& +\left\{\frac { \nu ( 1 + \nu ) } { ( 1 - \nu ) } \frac { \alpha _ { 2 } b } { K } T _ { a } \sum _ { m = 0 } ^ { \infty } \frac { \overline { f } ( s _ { m } ) } { s _ { m } ^ { 2 } } \left[D_{n} I_{0}^{\prime \prime}\left(s_{m} \rho b\right) s_{m}^{2}\right.\right. \\
& \left.+E_{n} s_{m}^{2}\left[\rho I_{1}^{\prime \prime}\left(s_{m} \rho b\right)+2 I_{1}^{\prime}\left(s_{m} \rho b\right)\right]\right] \cos \left(s_{m} \xi b\right) s_{m} b \\
& +\frac{1}{\rho} \frac{\nu(1+\nu)}{(1-\nu)} \frac{\alpha_{2}}{K} T_{a} \sum_{m=0}^{\infty} \frac{\bar{f}\left(s_{m}\right)}{s_{m}^{2}}\left[D_{n} I_{0}^{\prime}\left(s_{m} \rho b\right) s_{m}\right. \\
& \left.+E_{n} s_{m}\left[\rho I_{1}^{\prime}\left(s_{m} \rho b\right) s_{m} b+I_{1}\left(s_{m} \rho b\right)\right]\right] \cos \left(s_{m} \xi b\right) s_{m} b \\
& -\frac{\nu(1+\nu)}{(1-\nu)} \frac{\alpha_{2} b}{K} T_{a} \sum_{m=0}^{\infty} \bar{f}\left(s_{m}\right)\left[D_{n} I_{0}\left(s_{m} \rho b\right)\right. \\
& \left.+E_{n} s_{m}\left[\rho b I_{1}\left(s_{m} \rho b\right)\right]\right] \cos \left(s_{m} \xi b\right) s_{m} b \\
& -\frac{(1+\nu)}{(1-\nu)} \frac{\alpha_{2}}{K} T_{a} \sum_{m=0}^{\infty} \frac{\bar{f}\left(s_{m}\right)}{s_{m}^{2}}\left[D_{n} I_{0}^{\prime \prime}\left(s_{m} \rho b\right) s_{m}^{2} b^{2}\right. \\
& \left.+E_{n} s_{m}\left[\rho I_{1}^{\prime \prime}\left(s_{m} \rho b\right) s_{m}^{2} b^{2}+2 s_{m} b I_{1}^{\prime}\left(s_{m} \rho b\right)\right]\right] \cos \left(s_{m} \xi b\right) s_{m} \\
& -\frac{(1+\nu)}{(1-\nu)} \frac{\alpha_{2}}{K} T_{a} \sum_{m=0}^{\infty} \frac{\bar{f}\left(s_{m}\right)}{s_{m}^{2}}\left[D_{n} I_{0}^{\prime \prime}\left(s_{m} \rho b\right) s_{m}^{2} b^{2}\right. \\
& \left.+E_{n} s_{m}\left[\rho I_{1}^{\prime \prime}\left(s_{m} \rho b\right) s_{m}^{2} b^{2}+2 s_{m} b I_{1}^{\prime}\left(s_{m} \rho b\right)\right]\right] \cos \left(s_{m} \xi b\right) s_{m}, \\
& \frac{\bar{\rho} \bar{\xi}}{2 G}=\frac{-(1+\nu)}{(1-\nu)} \frac{\alpha_{2} b^{2}}{K} T_{a} \sum_{m=0}^{\infty} \frac{\bar{f}\left(s_{m}\right)}{I_{0}\left(s_{m} a\right)}\left[I_{0}^{\prime}\left(s_{m} \rho b\right) s_{m}\right. \\
& \left.-\sum_{n=1}^{\infty} \frac{A_{n}}{\alpha_{2}\left(s_{m}^{2}+\beta_{2 n}^{2}\right)}\left[B_{n}\left\{J_{0}^{\prime}\left(\beta_{2 n} \rho b\right)+C_{n} Y_{0}^{\prime}\left(\beta_{2 n} \rho b\right) \beta_{2 n}\right\}\right]\right] \\
& \times\left(t^{\prime}+\frac{e^{-\alpha_{2}\left(s_{m}^{2}+\beta_{2 n}^{2}\right) \frac{b^{2} t^{\prime}}{K}}}{\alpha_{2}\left(s_{m}^{2}+\beta_{2 n}^{2}\right) \frac{b^{2}}{K}}\right) \sin \left(s_{m} \xi b\right) s_{m}
\end{aligned}
$$




$$
\begin{aligned}
& +(1+\nu) T_{a} \frac{\alpha_{2}}{K}\left\{b \sum _ { m = 0 } ^ { \infty } \frac { \overline { f } ( s _ { m } ) } { s _ { m } ^ { 2 } } \left[D_{n} I_{0}^{\prime \prime \prime}\left(s_{m} \rho b\right) s_{m}^{3} b+\right.\right. \\
& +E_{n} s_{m}^{2}\left[\rho I_{1}^{\prime \prime \prime}\left(s_{m} \rho b\right) s_{m}^{3} b^{2}+I_{1}^{\prime \prime}\left(s_{m} \rho b\right) s_{m} b\right] \\
& \left.\left.+2 I_{1}^{\prime \prime}\left(s_{m} \rho b\right) s_{m} b\right]\right] \sin s_{m} \xi b \\
& +\frac{1}{\rho} \sum_{m=0}^{\infty} \frac{\bar{f}\left(s_{m}\right)}{s_{m}^{2}}\left[D_{n} I_{0}^{\prime \prime}\left(s_{m} \rho b\right) s_{m}^{2} b\right. \\
& \left.+E_{n} s_{m}\left[\rho I_{1}^{\prime \prime}\left(s_{m} \rho b\right) s_{m}^{2} b^{2}+\left\{I_{1}^{\prime}\left(s_{m} \rho b\right) s_{m} b\right\}\right]\right] \sin s_{m} \xi b \\
& -\frac{1}{\rho^{2}} \sum_{m=0}^{\infty} \frac{\bar{f}\left(s_{m}\right)}{s_{m}^{2}}\left[D_{n} I_{0}^{\prime}\left(s_{m} \rho b\right) s_{m}\right. \\
& \left.+E_{n} s_{m}\left[\rho I_{1}^{\prime}\left(s_{m} \rho b\right) s_{m} b+I_{1}\left(s_{m} \rho b\right)\right]\right] \sin s_{m} \xi b \\
& -b \sum_{m=0}^{\infty} \bar{f}\left(s_{m}\right)\left[D_{n} I_{0}^{\prime}\left(s_{m} \rho b\right) s_{m} b\right] \\
& \left.\left.+E_{n} s_{m} b\left[\rho I_{1}^{\prime}\left(s_{m} \rho b\right) s_{m} b+I_{1}\left(s_{m} \rho b\right)\right]\right] \sin s_{m} \xi b\right\} \\
& +\frac{(1+\nu)}{(1-\nu)} \frac{\alpha_{2} b}{K} T_{a}\left\{\sum _ { m = 0 } ^ { \infty } \frac { \overline { f } ( s _ { m } ) } { s _ { m } ^ { 2 } } \left[D_{n} I_{0}^{\prime}\left(s_{m} \rho b\right) s_{m} b\right.\right. \\
& \left.+E_{n} s_{m}\left[\rho I_{1}^{\prime}\left(s_{m} \rho b\right) s_{m} b+I_{1}\left(s_{m} \rho b\right)\right]\right] \sin \left(s_{m} \xi b\right) s_{m}^{2} .
\end{aligned}
$$

Similarly we can obtain the stresses $\sigma_{\xi \xi}$ and $\sigma_{\theta \theta}$.

\section{Numerical Results and Discussion}

Setting the following values for pure copper in both layers of equations (28) through (33), and solving them numerically and graphically, the patterns in the graphs have been analyzed and conclusions have been drawn accordingly:

$$
\begin{gathered}
h=0.1, \quad \alpha=16.5 \times 10^{-6}(\mathrm{~J} / \mathrm{K})=\alpha_{1}=\alpha_{2}, \\
\nu=0.35, \quad \lambda=386 \mathrm{~W} / \mathrm{m} / \mathrm{k}, \mathrm{G}=44.7 G P a, \\
K_{1}=K_{2}=117 x 10^{6}\left(\mathrm{~m}^{2} / \mathrm{s}\right), a=1.5, b=1.8, \\
\xi=-0.1 \cdots+1.1, \quad A_{n}=0.5, B_{n}=1.1, C_{n}=1.5, D_{n}=1.2, E_{n}=1.3 .
\end{gathered}
$$

$s_{m}$ are roots of $\cos (s z)=0, \beta_{1 n}$ are roots of $J_{0}\left(\beta_{1 n} a\right)=0$,

$\beta_{2 n}$ are roots of

$$
h_{2}\left(J_{0}\left(\beta_{2 n} b\right)\right)-K_{2} \beta_{2 n} J_{1}\left(\beta_{2 n} b\right)=0,
$$




$$
f(\xi)=H\left(\xi_{0}-|\xi|\right)
$$

for $\xi_{0}=1$ we take $\bar{f}\left(s_{m}\right)=0.1 \ldots 0.9$.

To examine the influence of heating on the behavior of thermoelastic problem of two region concentric cylinders with copper as its material, we have performed numerical calculations. For convenience we have used dimensionless quantities and numerical results are presented here for $-0.1 \leq \xi \leq 1.1, \rho=1.5$ for region 1 and $\rho=1.8$ for region 2 . In this case the function $f(\xi)$ is expressed as

$$
f(\xi)=H\left(\xi_{0}-|\xi|\right)
$$

for $\xi_{0}=1$.

According to numerical results, graphs are plotted for dimensionless temperature distribution, dimensionless stresses for both the regions. Figures 2 and 3 show temperature distribution for region 1 and region 2 in radial direction. In both the regions initially temperature increases in the vicinity of $\xi=0$, then gradually decreases with increasing values of $\xi$ while it shows variation with increasing values of $\rho$. This effect is observed due to factor $f(\xi)$ in $T_{a} f(\xi)$ expressed as Heaviside step function $f(\xi)=H\left(\xi_{0}-|\xi|\right)$ and the thermal contact at interface. Figures 4 and 5 show radial displacement for region 1 and region 2 in axial and radial direction. In region 1 , radial displacement in $\xi$ direction shows initial tension and then turns to compression as $\xi$ increases, while in region 2 it shows a sinusoidal pattern. This is due to convectional boundary condition at outer boundary. Radial displacement in both regions in radial direction increases with the increasing values of $\rho$ due to heat flux. Figures 6 and 7 show radial stress $\rho \rho / 2 G$ for region 1 and region 2 in axial directions. The figures show that the value of radial stresses in both the regions turns from negative to positive values in the vicinity of $\xi=0$ and gradually increases in increasing direction of $\xi$ and $\rho$. This is due to continuity of heat flux at the perfect thermal contact of two regions. Figures 8 and 9 show axial stresses for both the regions in axial $\xi$ direction. In region 1, axial stresses $\xi \xi / 2 G$ initially increases with high intensity and then gradually decreases while in region 2, axial stresses $\xi \xi / 2 G$ decrease to negative values. This occurs due to heat convection at the boundary of region 2 . 


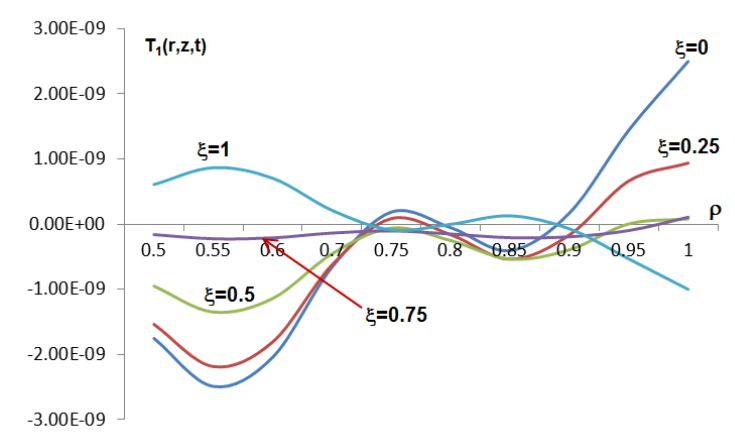

Figure 2: $T_{1}\left(\rho, \xi, t^{\prime}\right)$ versus $\rho$ for different values of $\xi$ in region 1

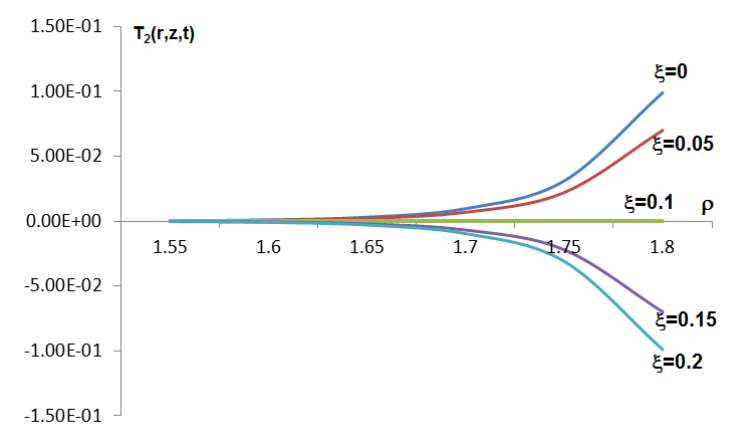

Figure 3: $T_{2}\left(\rho, \xi, t^{\prime}\right)$ versus $\rho$ for different values of $\xi$ in region 2

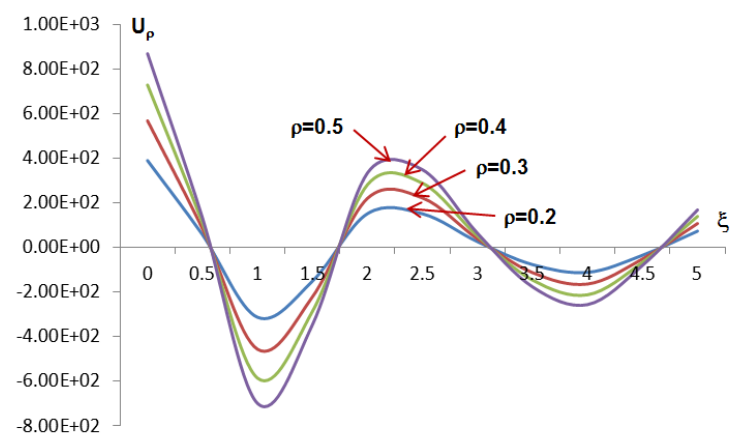

Figure 4: $U_{\rho}$ versus $\xi$ for different values of $\rho$ in region 1 


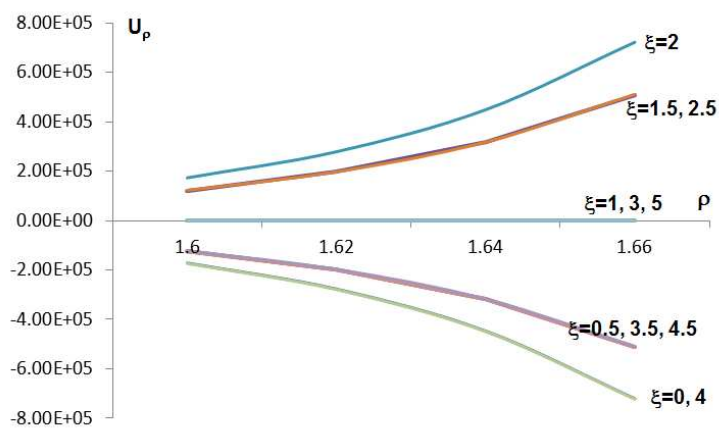

Figure 5: $U_{\rho}$ versus $\rho$ for different values of $\xi$ in region 2

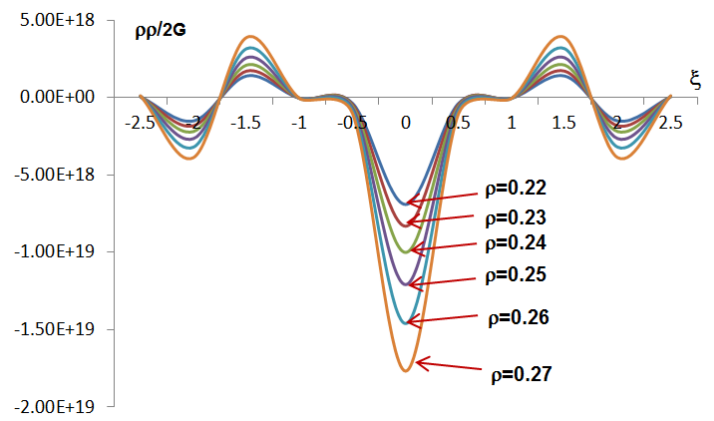

Figure 6: $\rho \rho / 2 \mathrm{G}$ versus $\xi$ for different values of $\rho$ in region 1

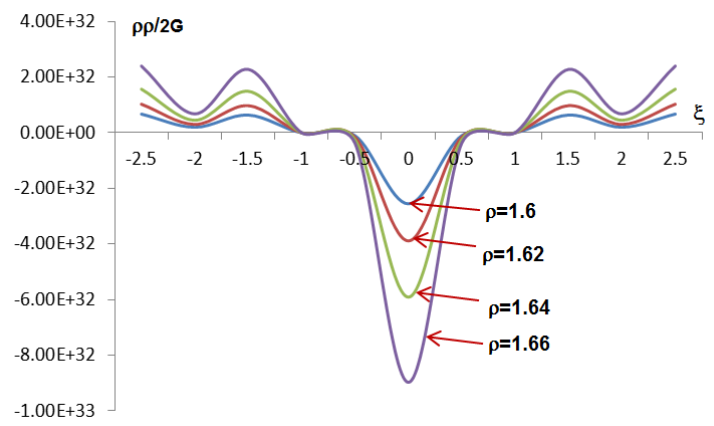

Figure 7: $\rho \rho / 2 \mathrm{G}$ versus $\xi$ for different values of $\rho$ in region 2 


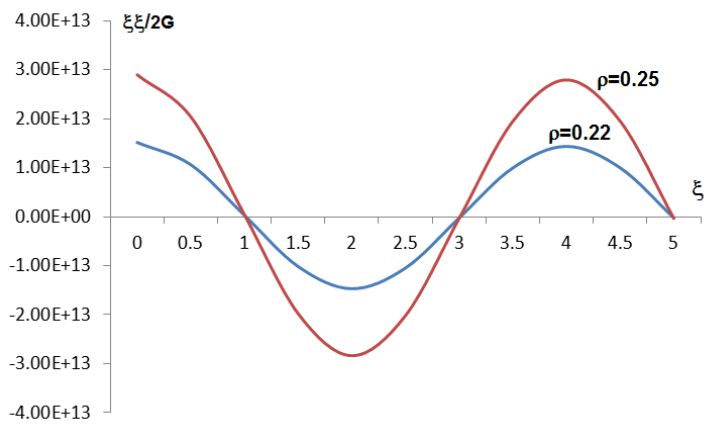

Figure 8: $\xi \xi / 2 \mathrm{G}$ versus $\xi$ for different values of $\rho$ in region 1

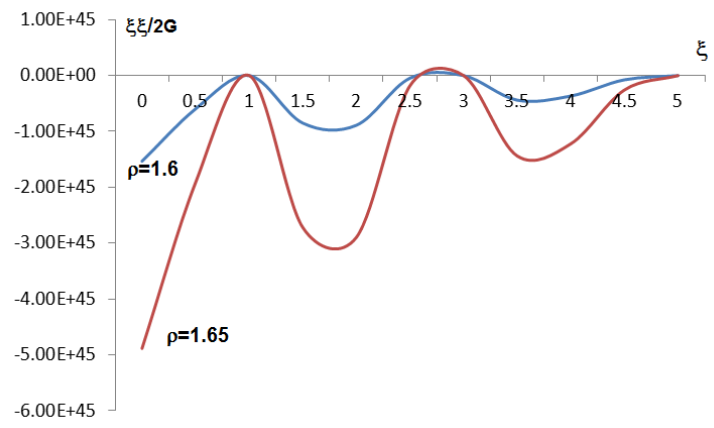

Figure 9: $\xi \xi / 2 \mathrm{G}$ versus $\xi$ for different values of $\rho$ in region 2

\section{References}

[1] G. Bonfanti, J.E. Muoz Rivera and M.G. Naso, Global existence and exponential stability for a contact problem between two thermoelastic beams, J. Math Anal. Appl., 345 (2008), 186-202.

[2] G. Bonfanti, M. Fabrizio, J.E. Muoz Rivera and M.G. Naso, On the energy decay for a thermoelastic contact problem involving heat transfer, $J$. Thermal Stresses, 33 (2010), 1049-1065.

[3] J.R. Barber, Introduction of the semi-infinite elastic solid by a hot sphere, Int. J. Mech. Sci., 15 (1973), 813-819.

[4] J.R. Barber, The effect of heat flow on the contact area between a contin- 
uous rigid punch and a frictionless elastic half space, J. Appl. Math. Phys., 27 (1976), 439-445.

[5] J.R. Barber, Indentation of an elastic half space, half space by a cooled flat punch, Q. J. Mech. Appl. Math, 35 (1982), 141-154.

[6] D.L. Elements and G.D. Toy, Two contact problems in anisotropic thermoelasticity, J. Elasticity, 6 (1976), 137-147.

[7] R.D. Kulchytsky-Zhyhailo, Z.S. Olesiak and O.O. Yevtushenko, On thermal contact of two axially symmetric elastic solids, Journal of Elasticity, 63 (2001), 1-17.

[8] Z.S. Olesiak, Axially symmetric thermal contact space, Bull. de l'Acad. Polon. Sci., Ser., 25 (1977), 163-166.

[9] H.H. Sherif and K.A. Helmy, A two dimensional problem for a half space in magnetothermoelasticity with thermal relaxation, Int. J. Eng. Sci. , 40 (2002), 587-604.

[10] O. Tamate, H. Sekine and T. Hanzawa, Thermal-stress singularities at the ends of a thin elastic stiffener bonded to a semi-infinite medium, J. Appl. Mech., 147 (1980), 436-438.

[11] A.A. Yevtushenko and R.D. Kulchytsky-Zhyhailo, Approximation solution and the thermoelastic contact problem with frictional heating the general case of the profile shape, J. of the Mechanics and Physics of Solids, 44 (1996), 243-250. 